\title{
Confiabilidade da informação sobre município de residência no Sistema de Informações Hospitalares - Sistema Único de Saúde para análise do fluxo de pacientes no atendimento do câncer de mama e do colo do útero
}

\section{Reliability of the information of the municipality of residence in the Hospital Information System of the Unified Health System for patients flow analysis for breast cancer and cervical cancer care}

Fernanda Pinheiro Aguiar1, Enirtes Caetano Prates Melo², Evangelina Xavier Gouveia de Oliveira ${ }^{2}$, Marilia Sá Carvalho ${ }^{3}$, Rejane Sobrino Pinheiro ${ }^{4}$

\begin{abstract}
Resumo
Objetivo: Analisar a qualidade do campo município de residência no Sistema de Informações Hospitalares do Sistema Único de Saúde (SIH-SUS). Metodologia: Foram selecionadas as internações de mulheres com diagnóstico principal de câncer de mama e de colo de útero e que morreram durante a internação em hospital do estado do Rio de Janeiro, 2001-2002. Estes registros foram comparados manualmente com os de óbito de residentes no estado do Rio de Janeiro, 2001-2002, considerados a referência para a comparação. Resultados: Dos 808 registros de óbitos hospitalares: 16 (1,9\%) não foram encontrados na base do Sistema de Informação sobre Mortalidade (SIM); em 670 (82,9\%) os municípios de residência coincidiram; 78 (9,6\%) indicavam município de residência Rio de Janeiro diferente do encontrado no SIM; e, destes, 53 (74,6\%) eram municípios da Região Metropolitana. O coeficiente kappa foi de 0,80 (IC95\% 0,76-0,84). Conclusão: Observou-se elevada concordância, menor quando a mulher não residia na capital, mas no seu entorno.
\end{abstract}

Palavras-chave: informação; estatísticas vitais; sistemas de informação hospitalar; mortalidade hospitalar; neoplasias de mama; neoplasias do colo do útero.

\footnotetext{
Abstract

Objective: To analyze the quality of the field municipality of residence in the Hospital Information System of the Unified Health System (SIH-SUS). Methods: We selected hospitalizations of women diagnosed with primary breast cancer and cervical cancer and who died during hospital admission in the state of Rio de Janeiro, 2001-2002. These records were compared manually with the death records of residents in the state of Rio de Janeiro, 2001-2002, considered the reference
}

\footnotetext{
Trabalho realizado no Instituto de Estudos em Saúde Coletiva (IESC) da Universidade Federal do Rio de Janeiro (UFRJ) - Rio de Janeiro (RJ), Brasil. ${ }^{1}$ Mestre em Saúde Coletiva pelo IESC da UFRJ - Rio de Janeiro (RJ), Brasil.

2Doutorado em Saúde Pública pela Escola Nacional de Saúde Pública da Fundação Oswaldo Cruz (FIOCRUZ); Pesquisadora do Grupo de Estudos em Redes e Informação para a Saúde da FIOCRUZ - Rio de Janeiro (RJ), Brasil.

${ }^{3}$ Doutorado em Engenharia Biomédica pela UFRJ; Pesquisadora do Grupo de Estudos em Redes e Informação para a Saúde da FIOCRUZ - Rio de Janeiro (RJ), Brasil. ${ }^{4}$ Doutorado em Saúde Pública pela Escola Nacional de Saúde Pública da FIOCRUZ. Professora Adjunta do Departamento de Medicina Preventiva da Faculdade de Medicina da UFRJ - Rio de Janeiro (RJ), Brasil.

Endereço para correspondência: Rejane Sobrino Pinheiro - Instituto de Estudos em Saúde Coletiva da Universidade Federal do Rio de Janeiro Avenida Horácio Macedo, s/n, Ilha do Fundão - Cidade Universitária - CEP: 21941-598 - Rio de Janeiro (RJ), Brasil - E-mail: rejane@iesc.ufrj.br Fonte de financiamento: Conselho Nacional de Desenvolvimento Científico e Tecnológico (CNPq) e Fundação de Amparo à Pesquisa do Estado do Rio de Janeiro (FAPERJ). Conflito de interesse: nada a declarar.
} 
for comparison. Results: Of 808 hospital deaths records: 16 (1.9\%) were not found in the Mortality Information System, in $670(82.9 \%)$ municipalities of residence coincided, 78 (9.6\%) indicated municipality of residence Rio de Janeiro, different from that found in the SIM, and, of these, 53 (74.6\%) were municipalities in the metropolitan region. The kappa coefficient was $0.80(95 \% \mathrm{Cl} 0.76-0.84)$. Conclusion: There was high overall agreement, lower when the woman did not live in the capital proper, but in its surroundings.

Keywords: information; vital statistics; hospital information systems; hospital mortality; breast neoplasms; uterine cervical neoplasms.

\section{INTRODUÇÃO}

No Sistema Único de Saúde (SUS), o atendimento oncológico é especializado e conta com uma rede de referência para o tratamento, distribuída em diferentes locais do território nacional.

A distribuição da oferta de procedimentos e serviços de saúde e a distância geográfica percorrida pelos indivíduos na busca de atendimento em saúde fornecem informação importante ao gestor para o monitoramento das ações implantadas, e sobre a necessidade de realocação dessa oferta ou de investimento de recursos na ampliação da mesma. Analisar os resultados da atenção à saúde, em função da distância ao local do atendimento, pode fornecer indicação sobre a qualidade da regionalização feita pelos serviços de referência, e as possíveis barreiras de acesso que estão sendo enfrentadas pelos indivíduos na busca de cuidado médico ${ }^{1-4}$.

Para a realização desses estudos, é fundamental a confiabilidade da informação sobre a localização da residência do paciente, cuja escala de análise no caso do atendimento de alta complexidade, como é o caso do oncológico, pode ser o nível municipal ${ }^{3,4}$.

No Brasil, diversos sistemas de informação em saúde registram o local de residência e de ocorrência do evento, seja ele óbito (Sistema de Informação sobre Mortalidade - SIM), internação (Sistema de Informações Hospitalares - SIHSUS) ou atendimento ambulatorial de quimioterapia e radioterapia (Sistema de Informação Ambulatorial de Alta Complexidade - SIA/APAC). Tais informações estão disponíveis no sítio do Datasus e podem dar origem a estudos sobre a organização espacial da oferta e sobre o fluxo do atendimento entre o local de residência e de atendimento.

No entanto, questiona-se que muitos pacientes informam equivocadamente como município de residência aquele no qual foram atendidos, por temer não receber o atendimento no local escolhido, dificultando conhecer as trajetórias dos pacientes até o local do atendimento. Já no caso do registro do óbito, crê-se que a informação do local de residência não sofra este viés.

O objetivo do presente trabalho foi analisar a qualidade da informação sobre município de residência no SIH-SUS, a partir do relacionamento e da comparação da informação contida no SIM, que será utilizado como padrão.

\section{MÉTODOS}

Foi realizado um estudo transversal a partir do relacionamento das bases de dados do SIH-SUS e do SIM. Foram selecionados os registros apresentados de mulheres que foram internadas com diagnóstico principal de câncer de mama e de colo de útero (Classificação Internacional de Doenças, 10 Revisão: C50, C53, D05 e D06) e morreram durante a internação, nos hospitais da rede SUS do estado do Rio de Janeiro, entre 2001 e 2002. Estes registros foram relacionados manualmente com os de óbito de residentes no estado do Rio de Janeiro, dos anos de 2001 e 2002.

Com o auxílio de mapas de fluxo, analisou-se a diferença entre o município registrado no SIH-SUS (destino) e o município registrado no SIM (origem), considerado nesse trabalho como a referência correta para o endereço de residência da mulher. Crê-se que haja menos erros no registro de endereço no SIM que no SIH-SUS, uma vez que, naquele, o indivíduo que foi a óbito deve apresentar um documento no momento do preenchimento da declaração de óbito, e os pacientes atendidos no SUS podem temer não receber $o$ atendimento se residirem fora do município onde a oferta está localizada, omitindo a informação correta. Para elaboração dos mapas de fluxo, foi utilizado o software Tabwin.

Foi calculado o índice kappa para a concordância entre os campos das diferentes bases de dados, usando o software Winpepi ${ }^{5}$.

O presente estudo foi aprovado pelo Comitê de Ética e Pesquisa do Instituto de Estudos em Saúde Coletiva/Universidade Federal do Rio de Janeiro, processo ${ }^{\circ}$ 06/2007.

\section{RESULTADOS}

Dos 808 registros de óbitos hospitalares do período estudado, $16(1,9 \%)$ não foram encontrados na base do SIM e $670(82,9 \%)$ dos municípios de residência coincidiram (Figuras 1 e 2). Setenta e oito (9,6\%) registros de internação indicavam município de residência Rio de Janeiro, diferentemente do SIM, o que correspondeu a $63,9 \%$ dos 122 casos de discordância. Destes 78 casos, 53 (74,6\%) residiam em municípios da Região Metropolitana. Já o contrário foi menos frequente: apenas $1,6 \%$ das internações de não residentes no município do Rio de Janeiro 
constavam no SIM como residindo na capital. Dos $53 \mathrm{mu}-$ nicípios com casos registrados no SIM (considerado, nesse trabalho, como os municípios que tiveram residentes que foram atendidas no SUS e que foram a óbito por câncer de mama ou de colo de útero), 24 deles tiveram casos de mulheres com registro de município de residência diferente, praticamente todos como Rio de Janeiro. Aproximadamente metade deles (14) pertence às Regiões Metropolitanas I e II.
Considerando a informação segundo a regional de saúde, o coeficiente kappa foi de 0,80 (IC95\% 0,76-0,84). Quando se levou em consideração a residência da mulher na capital ou fora dela, o coeficiente kappa foi de 0,77 (IC95\% 0,74-0,80), sugerindo que a maior parcela da discordância no campo município de residência entre as bases do SIH-SUS e a do SIM tenha ocorrido em favor da troca do município de residência original pelo Rio de Janeiro, local onde se concentra a maior quantidade de oferta de serviços de saúde.

\section{8}

Registros de óbito

hospitalar no SIH

\begin{tabular}{|c|c|c|}
\hline $\begin{array}{c}1,9 \%(16) \\
\text { Internações não foram } \\
\text { encontradas no SIM }\end{array}$ & $\begin{array}{c}82,9 \%(670) \\
\text { Municípios coincidiram } \\
\text { nas bases SIH e SIM }\end{array}$ & $\begin{array}{c}1,6 \%(13) \\
\text { Municípios de Residência } \\
\text { No SIH diferente de Rio de Janeiro } \\
\text { No SIM = Rio de Janeiro }\end{array}$ \\
\hline
\end{tabular}

\begin{tabular}{|c|}
$9,6 \%(78)$ \\
Municípios de Residência \\
No SIH = Rio de Janeiro \\
No SIM diferente de Rio de Janeiro
\end{tabular}

$74,6 \%(53)$

Municípios de Residência No SIM = Região Metropolitana

SIH: Sistema de Informações Hospitalares; SIM: Sistema de Informação sobre Mortalidade

Figura 1. Distribuição dos registros, segundo concordância ou não do município de residência, entre as bases de dados do Sistema de Informação sobre Mortalidade e do Sistema de Informações Hospitalares - Sistema Único de Saúde, 2001 e 2002

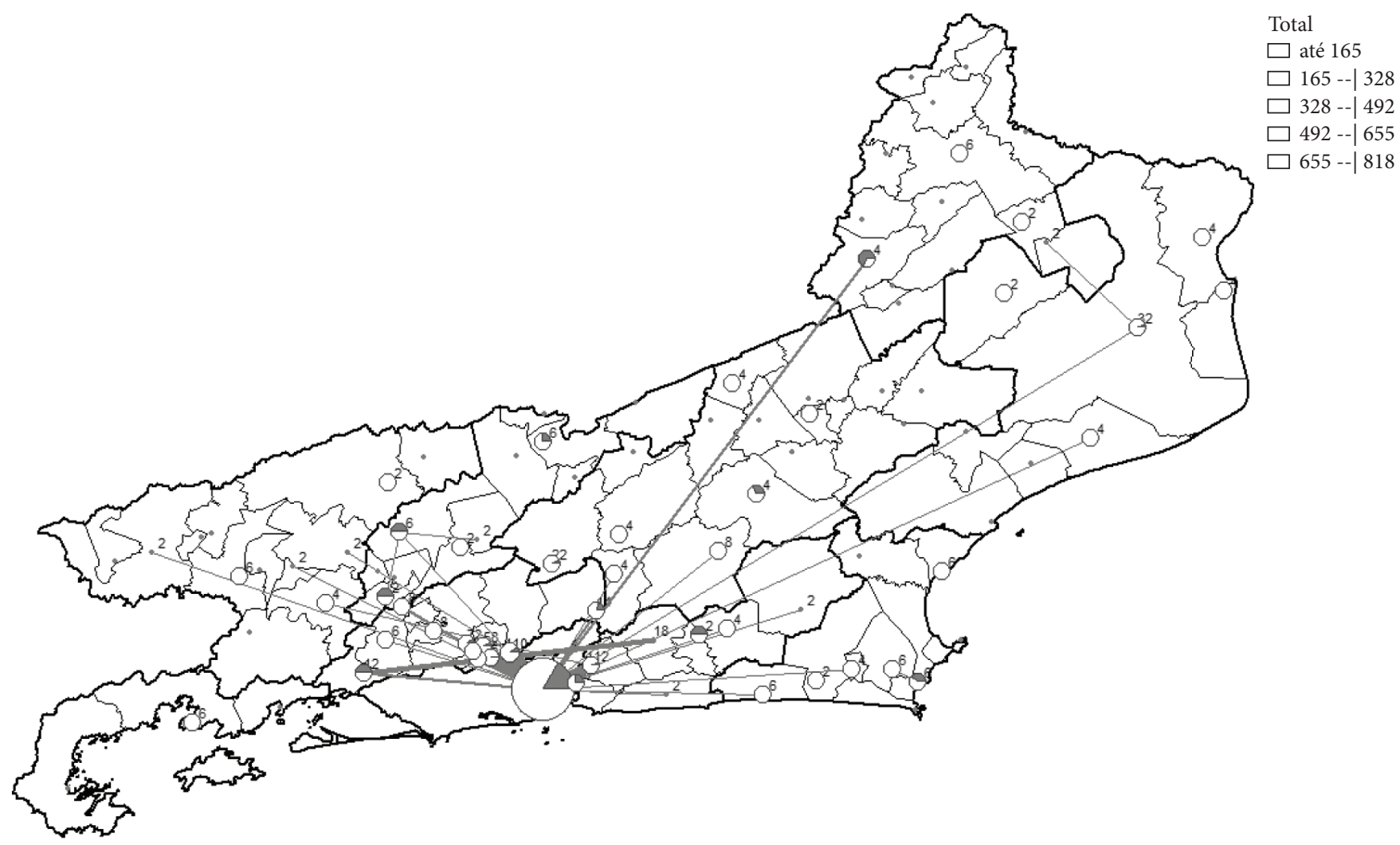

Figura 2. Mapa de fluxo cuja origem é o município registrado no Sistema de Informação sobre Mortalidade e o destino é o município registrado no Sistema de Informações Hospitalares - Sistema Único de Saúde 


\section{DISCUSSÃO}

Observou-se elevada concordância do município de residência entre o SIH-SUS e o SIM. Considerando o endereço no SIM como o endereço correto de residência da mulher para a análise dos casos discordantes, a maioria dos casos referia-se a mulheres que residem fora da capital, mas informam no atendimento o contrário ${ }^{6}$. Entretanto, proporção expressiva destes registros referia-se a mulheres que residiam no entorno do Rio de Janeiro, em sua Região Metropolitana, o que foi demonstrado também pelo elevado coeficiente kappa dentro da regional de saúde. A discordância pode ser atribuída ao receio da mulher de não receber o atendimento desejado em função de não residir no município onde busca a internação.

Os resultados aqui observados sugerem que os estudos de fluxo de pacientes entre local de residência e de atendimento, e os estudos de regionalização do atendimento com base no atendimento realizado contam com informação sobre o município de residência no SIH do SUS, com qualidade suficiente para sua execução e para apoiar o processo decisório na gestão em saúde.

Poucos registros de óbito hospitalar no SIH do SUS não foram encontrados no SIM. Alguns fatores podem explicar essa divergência nos resultados: pode ter ocorrido falha no processo de linkage, em função de preenchimento inadequado dos campos utilizados para comparação dos registros provenientes dos dois sistemas, dificultando o julgamento; o indivíduo que foi a óbito residia fora do estado do Rio de Janeiro e os seus dados não seriam encontrados na base de dados usada no linkage; sub-registro de óbito no SIM; erro de digitação ou de preenchimento do campo sobre o motivo de saída na internação que acusou o óbito; erro de preenchimento do município de residência no SIM. Possivelmente, uma combinação desses fatores resulta nas diferenças encontradas. Trabalhos mostram a elevada confiabilidade do registro de óbito no SIH-SUS ${ }^{7}$ e que, quando é registrado óbito como motivo da saída na internação, pequena é a chance de não encontrar o paciente no $\mathrm{SIM}^{8}$. Entretanto, a falha de linkage, nesse caso, não altera os resultados encontrados com relação à concordância do campo de endereço entre as bases. Ressalta, ainda, que o processo de relacionamento foi manual, com busca e comparação exaustiva dos registros, usando diversas variáveis entre os bancos para chegar-se à conclusão de que um par de registros referia-se ao mesmo indivíduo. Vale notar, finalmente, que os eventuais erros de linkage atuariam no sentido de reduzir a concordância observada do campo de endereço entre os dois sistemas analisados, pois, uma vez que pacientes distintos estariam sendo associados, isso aumentaria a chance de os municípios de residência serem diferentes.

\section{AGRADECIMENTOS}

Ao Conselho Nacional de Desenvolvimento Científico e Tecnológico (CNPq) è̀ Fundação de Amparo à Pesquisa do Estado do Rio de Janeiro (FAPERJ) pelo apoio financeiro à pesquisa.

\section{REFERÊNCIAS}

1. Skaba DA, Carvalho MS, Barcellos C, Martins PC, Terron SL. Geoprocessamento dos dados da saúde: o tratamento dos endereços. Cad Saúde Pública. 2004;20(6):1753-56.

2. Oliveira EXG, Melo ECP, Pinheiro RS, Noronha CP, Carvalho MS, Acesso à assistência oncológica: mapeamento dos fluxos origem-destino das internações e dos atendimentos ambulatoriais. O caso do câncer de mama. Cad Saúde Pública. 2011;27(2):317-26.

3. Oliveira EXG, Carvalho MS, Travassos C. Territórios do Sistema Único de Saúde mapeamento das redes de atenção hospitalar. Cad Saúde Pública. 2004;20(2):386-402.

4. Bousquat A, Nascimento VB. A Metrópole Paulista e a Saúde. São Paulo Perspec. 2001;15(1):112-20.

5. Abramson JH. WINPEPI (PEPI-for-Windows): computer programs for epidemiologists. Epidemiol Perspect \& Innov. 2004;1(1):6.
6. Costa LS, Pinheiro RS, Almeida RVR. Recuperação de informação de endereço na base de dados AIH: percentual de aproveitamento. Cad Saúde Coletiva. 2003;11:131- 41.

7. Veras CMT, Martins MS. A confiabilidade dos dados nos formulários de autorização de internação hospitalar (AIH), Rio de Janeiro, Brasil. Cad Saúde Púbica. 1994;10(3):339-55.

8. Pinheiro RS, Vieira RA, Coeli CM, Vidal EIO, Camargo Jr KR. Utilização do sih-sus e do sim para o cálculo da mortalidade hospitalar e em 30 dias para as internações de pacientes com fratura proximal de fêmur. Cad Saúde Coletiva. 2006;14(2):337-44.

Recebido em: 18/06/2012

Aprovado em: 22/05/2013 